\title{
A Novel Combination of Prion Strain Co-Occurrence in Patients with Sporadic Creutzfeldt-Jakob Disease
}

Atsushi Kobayashi, ${ }^{*}$ Yasushi Iwasaki, ${ }^{\dagger}$ Masaki Takao, ${ }^{\ddagger}$ Yuko Saito, ${ }^{\S}$ Toru Iwaki, ${ }^{\circledR}$ Zechen Qi, ${ }^{*}$ Ryouta Torimoto, ${ }^{*}$ Taishi Shimazaki, ${ }^{*}$ Yoshiko Munesue, ${ }^{*}$ Norikazu Isoda, ${ }^{* *}$ Hirofumi Sawa, ${ }^{\| \dagger \dagger}$ Keisuke Aoshima, ${ }^{*}$ Takashi Kimura, Hinako Kondo, ${ }^{\ddagger \ddagger}$ Shirou Mohri, ${ }^{\ddagger}$ and Tetsuyuki Kitamoto ${ }^{\ddagger \ddagger}$

From the Laboratory of Comparative Pathology, * Faculty of Veterinary Medicine, the Global Station for Zoonosis Control, "Global Institute for Collaborative Research and Education, the Unit of Risk Analysis and Management, ${ }^{* *}$ and the Division of Molecular Pathobiology, ${ }^{\dagger \dagger}$ Research Center for Zoonosis Control, Hokkaido University, Sapporo; the Department of Neuropathology, ${ }^{\dagger}$ Institute for Medical Science of Ageing, Aichi Medical University, Nagakute, Aichi; the Department of Neurology, ${ }^{\ddagger}$ Saitama Medical University International Medical Center, Saitama; the Department of Laboratory Medicine, ${ }^{\S}$ National Center of Neurology and Psychiatry Hospital, Kodaira; the Department of Neuropathology, "Graduate School of Medical Sciences, Kyushu University, Fukuoka; and the Department of Neurological Science, ${ }^{\ddagger \ddagger}$ Tohoku University Graduate School of Medicine, Sendai, Japan

Accepted for publication

February 15, 2019.

Address correspondence to Atsushi Kobayashi, Ph.D., D.V.M., Laboratory of Comparative Pathology, Faculty of Veterinary Medicine, Hokkaido University, Kita 18 Nishi 9, Kita-ku, Sapporo, 0600818, Japan. E-mail: kobayashi@vetmed.hokudai.ac. jp.
Six subgroups of sporadic Creutzfeldt-Jakob disease have been identified by distinctive clinicopathologic features, genotype at polymorphic codon 129 [methionine (M)/valine (V)] of the PRNP gene, and type of abnormal prion proteins (type 1 or 2). In addition to the pure subgroups, mixed neuropathologic features and the coexistence of two types of abnormal prion proteins in the same patient also have been reported. Here, we found that a portion of the patients previously diagnosed as MM1 had neuropathologic characteristics of the MM2 thalamic form (ie, neuronal loss of the inferior olivary nucleus of the medulla). Furthermore, coexistence of biochemical features of the MM2 thalamic form also was confirmed in the identified cases. In addition, in transmission experiments using prion protein-humanized mice, the brain material from the identified case showed weak infectivity and generated characteristic abnormal prion proteins in the inoculated mice resembling those after inoculation with brain material of MM2 thalamic form. Taken together, these results show that the co-occurrence of MM1 and MM2 thalamic form is a novel entity of sporadic Creutzfeldt-Jakob disease prion strain co-occurrence. The present study raises the possibility that the co-occurrence of the MM2 thalamic form might have been overlooked so far because of the scarcity of abnormal prion protein accumulation and restricted neuropathology. (Am J Pathol 2019, 189: 1276-1283; https://doi.org/10.1016/j.ajpath.2019.02.012)
Prion diseases are lethal transmissible neurodegenerative diseases including Creutzfeldt-Jakob disease (CJD), Gerstmann-Sträussler-Scheinker syndrome, fatal familial insomnia (FFI), and variably protease-sensitive prionopathy in humans, or scrapie, bovine spongiform encephalopathy, and chronic wasting disease in animals. The central event in the pathogenesis of prion diseases is a conformational conversion of the normal cellular isoform of prion protein into an abnormal misfolded isoform $\left(\mathrm{PrP}^{\mathrm{Sc}}\right)$, which is a component of a proteinaceous infectious particle, namely prion. ${ }^{1}$ The conformational conversion of the normal cellular isoform of prion protein can occur as a result of one of three causes: (i) spontaneous conversion in sporadic CJD
(sCJD) and variably protease-sensitive prionopathy; (ii) pathogenic mutations of the PRNP gene in genetic CJD, Gerstmann-Sträussler-Scheinker syndrome, and FFI; or (iii)

Supported by Japan Society for the Promotion of Science KAKENHI grants 18K05963 (A.K.) and 18K06506 (M.T.); a grant from the Ichiro Kanehara Foundation (A.K.); a grant from the Suhara Memorial Foundation (A.K.); a grant from The Kato Memorial Trust for Nambyo Research (A.K.); the Research Committee of Prion Disease and Slow Virus Infection, Research on Policy Planning and Evaluation for Rare and Intractable Diseases, Health and Labour Sciences Research grants, the Ministry of Health, Labour and Welfare, Japan (M.T.); and Japan Agency for Medical Research and Development grant JP18dm0107103 (Y.S.).

Disclosures: None declared. 
prion infection in acquired prion diseases such as iatrogenic CJD, kuru, variant CJD, scrapie, bovine spongiform encephalopathy, and chronic wasting disease. ${ }^{2}$

Patients with SCJD show clinical and neuropathologic heterogeneity associated with the genotype [methionine (M) or valine (V)] at polymorphic codon 129 of the PRNP gene and the type ( 1 or 2 ) of $\mathrm{PrP}^{\mathrm{Sc}}$ accumulating in the brain. ${ }^{3}$ The types 1 and $2 \mathrm{PrP}^{\mathrm{Sc}}$ can be distinguished by the size of proteinase $\mathrm{K}$-resistant core of the protein $(21$ and $19 \mathrm{kDa}$, respectively). ${ }^{3,4}$ Based on these two determinants, SCJD patients currently are classified into six subgroups: MM/MV1, MM2 cortical form (MM2C), MM2 thalamic form (MM2T), VV1, VV2, and MV2. ${ }^{5}$ The MM1 and MV1 subgroups are merged into one subgroup as MM/MV1 because they are indistinguishable in clinicopathologic and biochemical features. ${ }^{3} \mathrm{On}$ the other hand, the MM2 subgroup is divided further into two subgroups, MM2C and MM2T, because they show distinctive neuropathologic features. ${ }^{3}$ Widespread confluent vacuoles and intense perivacuolar PrP deposition in the cerebral cortices are characteristics of MM2C patients. By contrast, in MM2T patients, neuronal loss and gliosis are restricted within thalamic nuclei and the inferior olivary nucleus of the medulla, and PrP deposition is faint or negative. Transmission properties of the six SCJD subgroups also have been examined systematically, and five prion strains have been recognized based on the distinctive transmission properties, namely M1 (sCJDMM/MV1), M2C (sCJD-MM2C), M2T (sCJD-MM2T), V1 (sCJD-VV1), or V2 (sCJD-VV2 and -MV2) strain. ${ }^{6,7}$

Co-occurrence of different prion strains in the same brain is possible and results in the presentation of mixed neuropathologic features and more than one $\mathrm{PrP}^{\mathrm{Sc}}$ type. ${ }^{3,8-12}$ The most frequently observed mixed subgroup is co-occurrence of $\mathrm{M} 1$ and $\mathrm{M} 2 \mathrm{C}$ prion strains (ie, sCJD-MM/MV1+2C), which accounts for $26 \%$ of the total sCJD cases. ${ }^{12}$ The sCJD MM/MV1+2C patients show neuropathologic and biochemical features of sCJD MM/MV2C (ie, confluent vacuoles, perivacuolar $\mathrm{PrP}$ deposition, and type $2 \mathrm{PrP}^{\mathrm{Sc}}$ accumulation), besides sCJD-MM/MV1 characteristics. Moreover, coexisting M2C prion strain can affect the clinical features of patients as well as neuropathologic and biochemical properties, and the duration of illness becomes longer with increasing M2C prion strain load. ${ }^{11,12}$ However, a coexisting $\mathrm{M} 2 \mathrm{C}$ prion strain does not affect the transmission properties because the infectivity of the M2C strain is very low. ${ }^{13}$

The relatively high incidence of co-occurrence of M1 and M2C strains prompted us to investigate the possibility of other combinations of sCJD prion strain co-occurrence. To date, co-occurrence of V1 and V2 strains, M2C and V2 strains, M1 and V2 strains, or M2C and M2T strains has been reported, although the incidence rates are very low. ${ }^{12}$ Here, we show that a portion of SCJD cases previously diagnosed as MM1 had neuropathologic characteristics of sCJD MM2T. Furthermore, the coexistence of biochemical properties of sCJD MM2T also was confirmed in the identified cases. The present study suggests that the co- occurrence of M1 and M2T prion strains also may occur with a relatively high incidence rate.

\section{Materials and Methods}

\section{Ethics Statement}

This study was approved by the Institutional Ethics Committee of Hokkaido University Graduate School of Veterinary Medicine (VET27-1). All experiments using human materials were in compliance with the Helsinki Declaration. Animal experiments were performed in strict accordance with the Regulations for Animal Experiments and Related Activities at Hokkaido University and Fundamental Guidelines for Proper Conduct of Animal Experiment and Related Activities in Academic Research Institutions by the Ministry of Education, Culture, Sports, Science and Technology in Japan notice no. 71. The protocol was approved by the Institutional Animal Care and Use Committees of Hokkaido University (14-0170).

\section{Patients}

CJD cases included in this study were patients with clinically, genetically, and histopathologically proven sCJD, genetic CJD, and FFI. Brain tissues were obtained at autopsy from CJD patients after receiving written informed consent for research use. The diagnosis of CJD, histopathologic type, and $\mathrm{PrP}^{\mathrm{Sc}}$ type were confirmed by PrP immunohistochemistry and Western blot analysis. The genotype and mutations in the open reading frame of the PRNP gene were determined by sequence analysis as described. ${ }^{14}$ According to the classification by Parchi et al, ${ }^{5}$ the CJD cases were classified as follows: sCJD MM1, 18 cases; sCJD MM1+2C, 9 cases; sCJD MM2C, 3 cases; sCJD MM2T, 4 cases; sCJD MV2, 3 cases; genetic CJD V180I, 9 cases; genetic CJD E200K, 2 cases; and FFI, 5 cases. Detailed clinicopathologic features of one sCJD MM1+2C patient (H186) with neuronal loss of the inferior olivary nucleus have been reported elsewhere. ${ }^{15}$

\section{Histopathologic Analysis}

Formalin-fixed brain tissues were treated with formic acid (99\% for human tissues or $60 \%$ for mouse tissues) for 1 hour to inactivate the infectivity and embedded in paraffin. The embedded tissues were sectioned at a thickness of 5 $\mu \mathrm{m}$. For PrP immunohistochemistry, tissue sections were pretreated by hydrolytic autoclaving. ${ }^{16}$ The anti-PrP antiserum PrP-N ${ }^{17}$ was used as the primary antibody. Goat antirabbit immunoglobulin polyclonal antibody labeled with the peroxidase-conjugated dextran polymer, EnVision ${ }^{+}$(Dako, Glostrup, Denmark), was used as the secondary antibody. For routine histopathologic analysis, the tissue sections were stained with hematoxylin and eosin. For quantification of neuronal loss in the inferior olivary nucleus, the tissue 
sections of the medulla were stained by the Kluver-Barrera $^{18}$ method, and the remaining neurons in the right and left inferior olivary nuclei were counted. The number of the remaining neurons was divided by the area of the inferior olivary nucleus, which was measured by ImageJ software version 1.52a (NIH, Bethesda, MD; http://rsb.info.nih. gov/ij), and the neuronal cell density was calculated.

\section{PrP ${ }^{S c}$ Purification}

$\operatorname{PrP}^{\mathrm{Sc}}$ was purified from human brains or mouse brains as described. ${ }^{19}$ Briefly, brain tissues were homogenized in 2 $\mathrm{mL}$ of lysis buffer $(100 \mathrm{mmol} / \mathrm{L}$ Tris- $\mathrm{HCl} \mathrm{pH} \mathrm{8.0,} 10$ $\mathrm{mmol} / \mathrm{L} \mathrm{NaCl}, 10 \mathrm{mmol} / \mathrm{L} \mathrm{MgCl} 2,2 \%$ Triton X-100, and 25 $\mathrm{U} / \mathrm{mL}$ DNase I; all from Takara Bio, Shiga, Japan) and digested with collagenase $(1 \mathrm{mg} / 200 \mathrm{mg}$ tissue; FUJIFILM Wako Pure Chemical Corporation, Osaka, Japan) overnight at room temperature. Collagenase digestion disrupts the connective tissue and improves the accessibility of detergents and/or proteinase $\mathrm{K}$ to $\operatorname{PrP}^{\mathrm{Sc}} \cdot{ }^{20}$ The digested homogenates were ultracentrifuged at $453,000 \times g$ for 30 minutes at $4{ }^{\circ} \mathrm{C}$, and the pellets were resuspended and sonicated in $870 \mu \mathrm{L}$ of proteinase $\mathrm{K}$-digestion buffer $(100$ $\mathrm{mmol} / \mathrm{L}$ Tris-HCl $\mathrm{pH} 8.0$ and 5\% Sarkosyl; Sigma-Aldrich, St. Louis, MO). The resuspended samples were centrifuged at $1000 \times g$ for 3 minutes to remove the cell debris, and the supernatants $(800 \mu \mathrm{L})$ were digested with proteinase $\mathrm{K}(4$ $\mu \mathrm{g} / 200 \mathrm{mg}$ tissue) (FUJIFILM Wako Pure Chemical Corporation) for 1 hour at $37^{\circ} \mathrm{C}$. It has been reported that these conditions for proteinase $\mathrm{K}$ digestion were sufficient for the complete digestion of the normal cellular isoform of prion protein, and that higher proteinase $\mathrm{K}$ concentrations caused unfavorable degradation of $\mathrm{PrP}^{\mathrm{Sc}} \cdot{ }^{21}$ The proteinase $\mathrm{K}$-digested proteins were precipitated by adding $200 \mu \mathrm{L}$ of $99.5 \%$ ethanol and ultracentrifugation at $135,000 \times g$ for 30 minutes at $4{ }^{\circ} \mathrm{C}$. The pellets were resuspended in $400 \mu \mathrm{L} / 200$ $\mathrm{mg}$ tissue of Laemmli's sample buffer $(60 \mathrm{mmol} / \mathrm{L}$ Tris-HCl $\mathrm{pH} 6.8,5 \%$ glycerol, $2 \%$ SDS, and $0.01 \%$ bromophenol blue) and boiled at $100^{\circ} \mathrm{C}$ for 10 minutes.

\section{Western Blot Analysis}

Protein samples were subjected to SDS-PAGE using 13.5\% Bis-Tris long gels of $15 \mathrm{~cm}$ length and Western blot as described. $^{22}$ The anti-PrP monoclonal antibody 3F4 (BioLegend, San Diego, CA) and type $2 \mathrm{PrP}^{\mathrm{Sc}}$-specific antiPrP polyclonal antibody Tohoku $2^{23}$ were used as the primary antibodies. The anti-mouse EnVision+ (Dako) and anti-rabbit EnVision+ (Dako) were used as the secondary antibodies. The blots were visualized with Clarity Max Western ECL substrate (Bio-Rad, Hercules, CA), and images were obtained by the imaging device ImageQuant LAS 4000 mini (GE Healthcare, Chicago, IL). The signal intensities of the Western blots were quantified with ImageQuant TL software version 7.0 (GE Healthcare).

\section{Transmission Experiments}

The production of knock-in mice expressing human PrP with the $129 \mathrm{M} / \mathrm{M}$ genotype $(\mathrm{Ki}-\mathrm{Hu} 129 \mathrm{M} / \mathrm{M})$ has been reported previously. ${ }^{22}$ Knock-in mice expressing human PrP carrying a causative mutation of FFI (aspartic acid to asparagine at codon $178, \mathrm{Ki}-\mathrm{HuD} 178 \mathrm{~N}$ ) were generated as described. ${ }^{24}$ The codon 129 genotype of the Ki-HuD178N mice was $129 \mathrm{M} / \mathrm{M}$. Intracerebral inoculation was performed as described. ${ }^{25}$ Briefly, $10 \%$ brain homogenates were prepared in sterile phosphate-buffered saline using glass homogenizers, and $20 \mu \mathrm{L}$ of the homogenates were inoculated intracerebrally into mice. The inoculated mice were sacrificed at a predefined clinical end point, or at the time point showing intercurrent illness. One hemisphere of the brain was fixed in $10 \%$ buffered formalin for histopathologic analysis, and the other hemisphere was immediately frozen for biochemical analysis.

\section{Statistical Analysis}

Signal intensities of $\mathrm{PrP}^{\mathrm{Sc}}$ bands are expressed as means $\pm \operatorname{SEM}(n=3)$. The Kaplan-Meier log-rank test was used to analyze survival data in a transmission study. The statistical tests were performed using the statistical software EZR version 1.36 (Jichi Medical University, Saitama, Japan; http://www.jichi.ac.jp/saitama-sct/SaitamaHP.files/ statmed.html). ${ }^{26}$

\section{Results}

\section{Coexistence of Neuropathologic Characteristics of sCJD-MM2T}

Neuronal loss in the thalamus and the inferior olivary nucleus of the medulla is a key neuropathologic feature of sCJD-MM2T and FFI. ${ }^{3,27}$ Because thalamic nuclei also are affected in other CJD subgroups (Supplemental Figure S1), neuronal loss of the inferior olivary nucleus was studied to identify a coexisting M2T prion strain. Systematic neuropathologic analysis of archived prion disease tissue samples showed that 3 of the 18 (17\%) sCJD MM1 patients showed neuronal loss of the inferior olivary nucleus, as with SCJD MM2T or FFI patients (Figure 1). Other neuropathologic changes were typical of SCJD MM1 such as neuronal loss, spongiform changes, gliosis, and diffuse synaptic-type $\operatorname{PrP}$ deposition in the cerebral and cerebellar cortices. Neuropathologic features of sCJD MM2C (eg, confluent vacuoles or perivacuolar PrP deposition), were not observed in these sCJD MM1 patients. In addition, three of nine (33\%) sCJD $\mathrm{MM} 1+2 \mathrm{C}$ patients also showed neuronal loss of the inferior olivary nucleus (Figure 1).

Although the sCJD MM1 patients with the inferior olivary degeneration showed a prolonged duration of illness (ie, duration from onset to death) (Table 1), a long clinical course was not solely responsible for the inferior olivary 


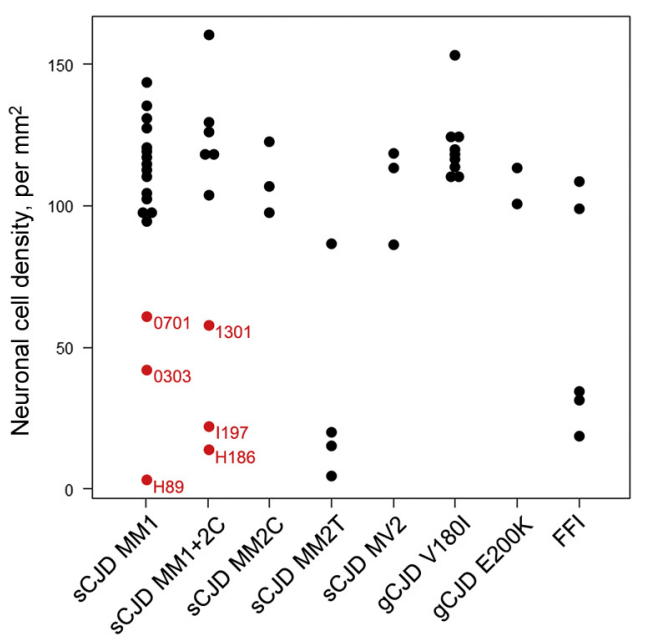

Figure 1 Neuronal loss of the inferior olivary nucleus of the medulla in Creutzfeldt-Jakob disease (CJD) patients. A portion of sporadic CreutzfeldtJakob disease (sCJD) patients previously diagnosed as MM1 (0701, 0303, and $\mathrm{H} 89)$ or MM1+2C (1301, I197, and H186) showed decreased neuronal cell density, similar to SCJD MM2T patients or fatal familial insomnia (FFI) patients. Black and red circles represent patients; SCJD MM1 or MM1+2C patients with neuronal loss of the inferior olivary nucleus are indicated by red circles. gCJD, genetic Creutzfeldt-Jakob disease.

degeneration because sCJD MM1 patients with a prolonged clinical course did not always show neuronal loss of the inferior olivary nucleus (Figure 2). Thus, some sCJD patients previously diagnosed as MM1 or $\mathrm{MM} 1+2 \mathrm{C}$ had neuropathologic characteristics of SCJD MM2T.

\section{Coexistence of Biochemical Features of sCJD MM2T}

To identify a coexisting M2T prion strain biochemically, Western blot analysis of proteinase $\mathrm{K}$-resistant $\mathrm{PrP}^{\mathrm{Sc}}$ was performed in the brain of the SCJD MM1 patients with the inferior olivary degeneration. Among the three sCJD MM1

Table 1 Summary of Clinical Features

\begin{tabular}{llll}
\hline Characteristics & $\begin{array}{l}\text { sCJD MM1 } \\
(\text { H89) }\end{array}$ & $\begin{array}{l}\text { sCJD MM1 } \\
(0303)\end{array}$ & $\begin{array}{l}\text { sCJD MM1 } \\
(0701)\end{array}$ \\
\hline Sex & Male & Male & Female \\
Age at onset, years & 61 & 63 & 71 \\
Initial symptoms & Progressive & Progressive & Progressive \\
& dementia & dementia & dementia \\
Myoclonus, months* & 15 & 8 & 3 \\
Akinetic mutism, months* & 29 & $12^{\dagger}$ & 6 \\
PSWC on EEG, months* & 15 & $-\ddagger$ & 2 \\
Duration, months & 36 & 24 & 24 \\
\hline
\end{tabular}

*The duration until the appearance of myoclonus, akinetic mutism, or PSWC from onset.

†The patient became bedridden seven months after the initial symptoms.

${ }^{\ddagger}$ Only a single EEG examination was performed 2 months after the initial symptoms. EEG revealed a short burst of delta waves and slowing of background activities.

EEG, electroencephalogram; PSWC, periodic sharp wave complexes; sCJD, sporadic Creutzfeldt-Jakob disease.

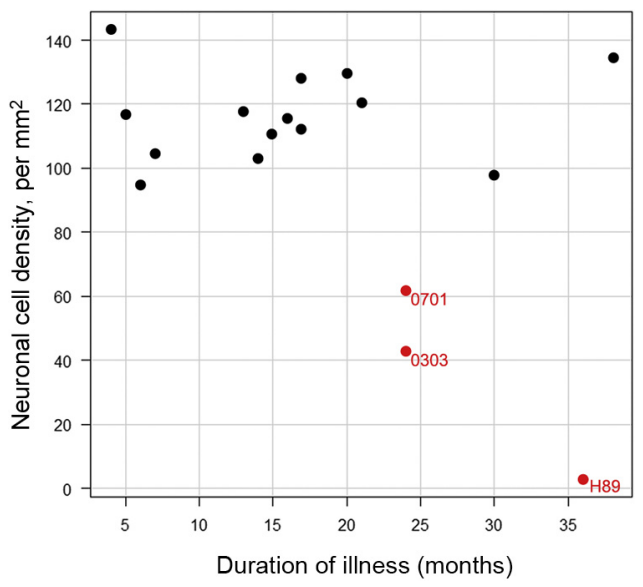

Figure 2 Neuronal loss of the inferior olivary nucleus does not depend on the duration of illness. Data are represented as the neuronal cell density of the inferior olivary nucleus of sporadic Creutzfeldt-Jakob disease (sCJD) MM1 patients ( $y$ axis) plotted against the duration of illness ( $x$ axis). Black and red circles represent patients; sCJD MM1 patients with neuronal loss of the inferior olivary nucleus are indicated by red circles.

patients with the inferior olivary degeneration, frozen brain tissues from multiple brain regions were available in two patients (H89 and 0303). In both patients, faint type $2 \mathrm{PrP}^{\mathrm{Sc}}$ bands (approximately $19 \mathrm{kDa}$ ) were visible alongside type 1 $\mathrm{PrP}^{\mathrm{Sc}}$ (approximately $21 \mathrm{kDa}$ ) in at least one brain region in Western blot analysis using the conventional anti-PrP antibody $3 \mathrm{~F} 4$ (Figure 3 ). By contrast, no type $2 \mathrm{PrP}^{\mathrm{Sc}}$ band was detected in a typical SCJD MM1 case lacking the inferior olivary degeneration (Supplemental Figure S2). Moreover, the type $2 \mathrm{PrP}^{\mathrm{Sc}}$-specific antibody Tohoku $2^{23}$ showed that small amounts of type $2 \mathrm{PrP}^{\mathrm{Sc}}$ were widely distributed throughout the brain except in the cerebellum of the two sCJD MM1 patients with the inferior olivary degeneration. Thus, although the previous examination using only a single brain region had shown only type $1 \mathrm{PrP}^{\mathrm{Sc}}$, re-examination using multiple brain regions identified coexisting type 2 $\mathrm{PrP}^{\mathrm{Sc}}$ in the SCJD patients previously diagnosed as MM1. Therefore, these patients also had biochemical features of sCJD MM2T in addition to neuropathologic characteristics. The sCJD MM1+2C patients with the inferior olivary degeneration (H186 and I197) had been previously examined biochemically, and both types 1 and $2 \mathrm{PrP}^{\mathrm{Sc}}$ were detected in the brain (Figure 4). ${ }^{15}$

\section{Coexistence of Transmission Properties of SCJD MM2T}

To identify a coexisting M2T prion strain based on transmission properties, a transmission study was performed using brain homogenates of sCJD MM1 or MM1 $2 \mathrm{C}$ patients with the inferior olivary degeneration. The inocula were prepared from brain regions where both types 1 and $2 \operatorname{PrP}^{\mathrm{Sc}}$ coexisted. In the sCJD MM1 $+2 \mathrm{C}$ patient, more than one brain region was used for inoculum preparation to enhance the chance of detecting a concurrent M2T prion strain because M2T and M2C prion strains are indistinguishable by Western blot 
A

3F4

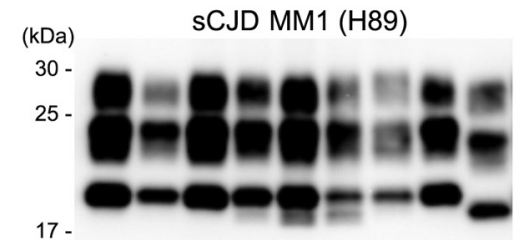

B

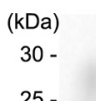

Tohoku 2

$25-$

17 -

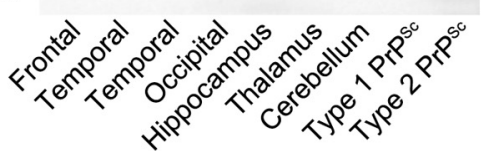

sCJD MM1 (0303)

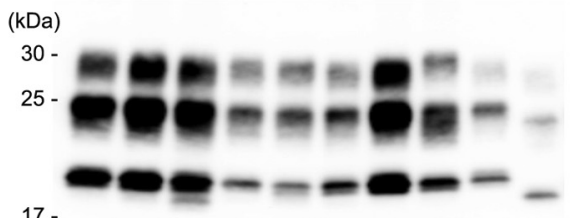

17 -

(kDa)

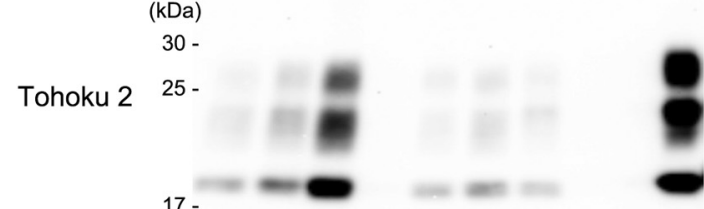

17 -

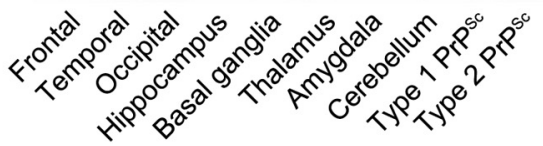

sCJD MM1 (H89)
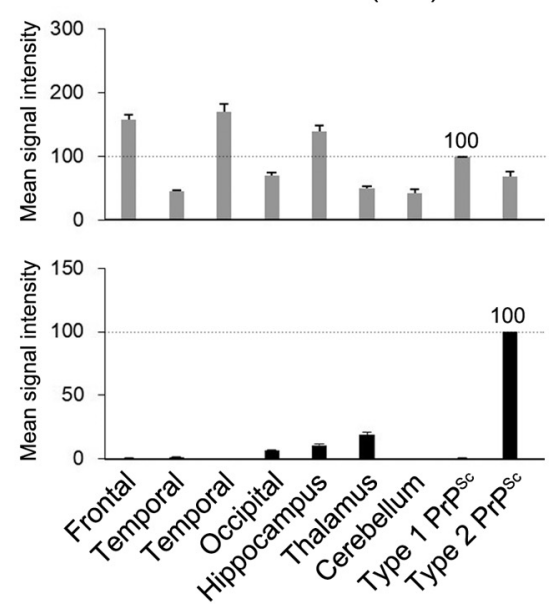

sCJD MM1 (0303)
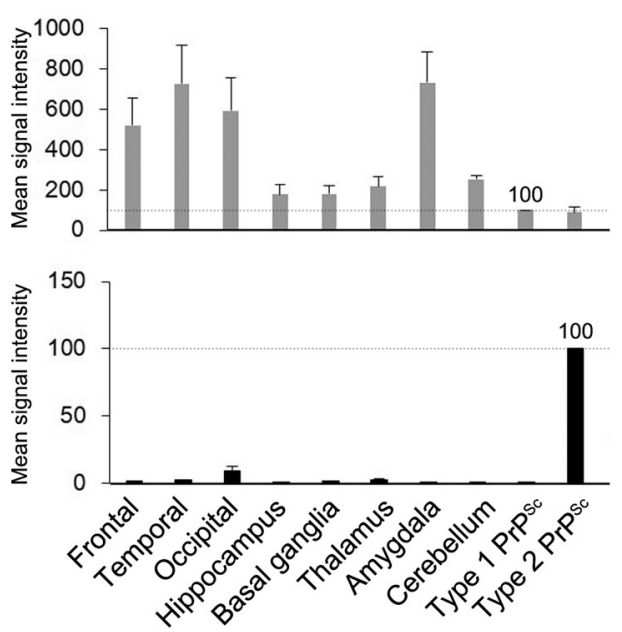

Figure 3 Type 2 abnormal misfolded isoform of the prion protein $\left(\mathrm{PrP}^{\mathrm{Sc}}\right)$ accumulation in the sporadic Creutzfeldt-Jakob disease (sCJD) MM1 patients with neuronal loss of the inferior olivary nucleus. A and B: Multiple brain regions of the SCJD MM1 patients were examined by Western blot using anti-PrP antibody $3 \mathrm{~F} 4$ or type $2 \mathrm{PrP}^{\mathrm{Sc}}$-specific antibody Tohoku 2. Type $2 \mathrm{PrP}^{\mathrm{Sc}}$ was relatively abundant in the occipital lobe (occipital), a mixture of the hippocampus and parahippocampus (hippocampus), and thalamus in one patient (H89) (A), whereas it was prominent only in the occipital lobe in the other patient (0303) (B). A brain sample, equivalent to $0.5 \mathrm{mg}$ in wet weight, was loaded in each lane. The mean signal intensities of $\operatorname{PrP}{ }^{\mathrm{Sc}}$ in type $1 \mathrm{PrP}^{\mathrm{Sc}}$ control (sCJD MM1) and type 2 $\mathrm{PrP}^{\mathrm{SC}}$ control (sCJD MM2T) were assigned as $100 / \mathrm{mm}^{2}$ in each experiment using the $3 \mathrm{~F} 4$ antibody (gray bars) and Tohoku 2 antibody (black bars), respectively. The signal intensities of $\operatorname{PrP}^{\mathrm{Sc}}$ are expressed as means \pm SEM. $n=3$.

analysis. The brain homogenates were inoculated intracerebrally into knock-in mice expressing human PrP with the $129 \mathrm{M} / \mathrm{M}$ genotype (Ki-Hu129M/M) or knock-in mice expressing human PrP carrying a causative mutation of FFI (Ki-HuD178N). The transmission patterns of one sCJD MM1 material (occipital lobe of 0303) and sCJD MM1+2C materials [thalamus (I197 Th) or a mixture of the hippocampus and parahippocampus of I197 (I197 Hip)] were identical to those of a typical sCJD MM1 material. Briefly, the mean incubation period (means $\pm \mathrm{SD}$ ) was shorter in Ki-Hu129M/ $\mathrm{M}$ mice compared with Ki-HuD178N mice [584 \pm 3 days versus $789 \pm 82$ days for sCJD MM1 (0303), $P<0.005$; $554 \pm 26$ days versus $653 \pm 56$ days for sCJD MM1 $12 \mathrm{C}$ (I197 Th), $P<0.005$; and $597 \pm 4$ days versus $703 \pm 55$ days for sCJD MM1+2C (I197 Hip)], $P<0.005$ (Figure 5, A-D). In addition, the inoculated $\mathrm{Ki}-\mathrm{Hu} 129 \mathrm{M} / \mathrm{M}$ mice produced a large amount of type $1 \mathrm{PrP}^{\mathrm{Sc}}$ whereas $\mathrm{Ki}$ HuD178N mice produced faint type $2 \mathrm{PrP}^{\mathrm{Sc}}$ (Figure 6). By contrast, the transmission patterns of the other SCJD MM1 material (thalamus of H89) was quite different from those of typical SCJD MM1. Briefly, the infectivity was weak regardless of the mouse genotype (Figure 5, E-G), and the inoculated $\mathrm{Ki}-\mathrm{Hu} 129 \mathrm{M} / \mathrm{M}$ mice produced faint type $1 \mathrm{PrP}^{\mathrm{Sc}}$ whereas Ki-HuD178N mice produced a di- and monoglycosylated form-dominant type $2 \mathrm{PrP}^{\mathrm{Sc}}$ (Figure 6). These transmission properties were similar to those of typical SCJD MM2T materials.

\section{Discussion}

Herein, we identified concurrence of characteristic features of sCJD MM2T, for example, neuronal loss of the inferior 


\section{sCJD MM1+2C (I197)}

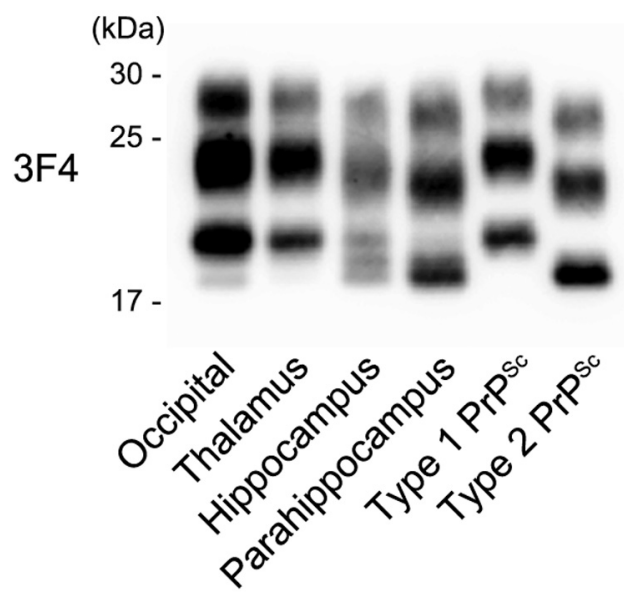

Figure 4 Type 2 abnormal misfolded isoform of the prion protein $\left(\mathrm{PrP}^{\mathrm{Sc}}\right)$ accumulation in the sporadic Creutzfeldt-Jakob disease (sCJD) $M M 1+2 C$ patients with neuronal loss of the inferior olivary nucleus. Type 2 $\mathrm{PrP}^{\mathrm{Sc}}$ is detected in the occipital lobe, hippocampus, and parahippocampus, although it is indistinguishable whether the detected type $2 \mathrm{PrP}^{\mathrm{Sc}}$ is an M2T prion strain or $\mathrm{M} 2 \mathrm{C}$ prion strain. A brain sample, equivalent to $1 \mathrm{mg}$ in wet weight, was loaded in each lane.

olivary nucleus of the medulla, type $2 \operatorname{PrP}^{\mathrm{Sc}}$ accumulation, and unique transmission properties, in SCJD patients previously diagnosed as MM1. The present study clearly shows that co-occurrence of M1 and M2T sCJD prion strains in the same patient also may occur.
The incidence rate of co-occurrence of M1 and M2T prion strains may be relatively high. In the present study, 3 of 18 sCJD cases previously diagnosed as MM1 had neuropathologic characteristics of sCJD-MM2T. The coexistence of more than one prion strain in the same patient accounted for $35 \%$ of total sCJD cases. ${ }^{12}$ Therefore, the present study, together with previous findings, ${ }^{12}$ suggests that co-occurrence of multiple prion strains is a more common phenomenon than expected. Indeed, concurrence of an M2T prion strain also was suggested in SCJD $\mathrm{MM} 1+2 \mathrm{C}$ patients in the present study. Because faint accumulation of $\operatorname{PrP}^{\mathrm{Sc}}$ and limited pathologic changes are characteristics of the M2T prion strain, ${ }^{3}$ coexistence of an $\mathrm{M} 2 \mathrm{~T}$ prion strain may have been overlooked to date. To estimate the exact prevalence of co-occurrence of an M2T prion strain, further analysis will be needed in the future with a larger number of patients. For this aim, the examination of neuronal loss of the inferior olivary nucleus can be one of the sensitive methods to identify a coexisting M2T prion strain.

The coexisting M2T prion strain may affect transmission properties of SCJD. A coexisting M2C prion strain does not affect transmission properties of an $\mathrm{M} 1$ prion strain in mice inoculated with brain materials from sCJD MM/MV1+2C patients. ${ }^{13}$ This is because the infectivity of the M2C prion strain is very low. ${ }^{6,13}$ Therefore, one can consider that the risk of transmission of an $\mathrm{M} 2 \mathrm{C}$ prion strain from SCJD MM/ $\mathrm{MV} 1+2 \mathrm{C}$ patients is negligible. By contrast, an M2T prion
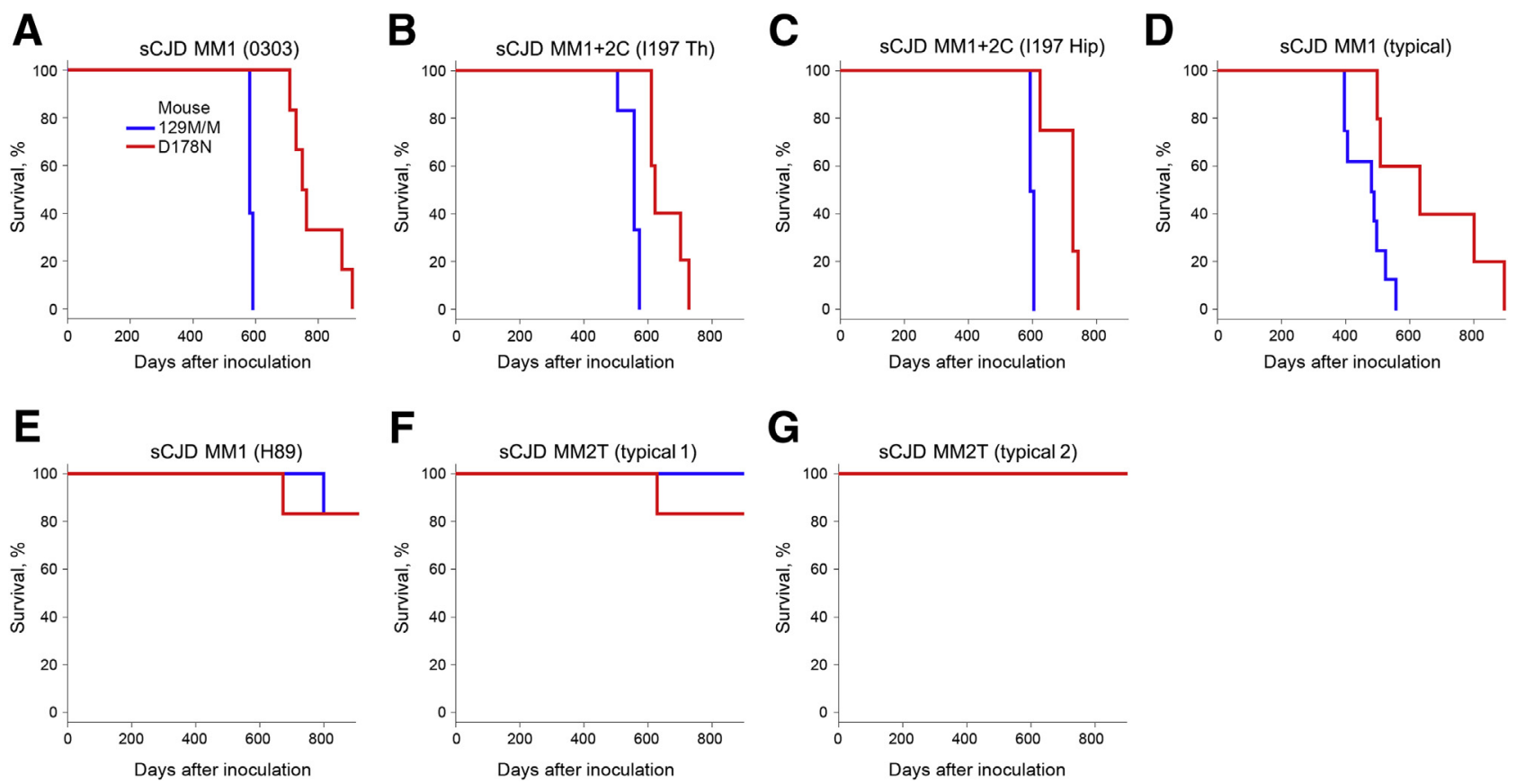

Figure 5 Kaplan-Meier survival curves after intracerebral inoculation into Ki-Hu129M/M mice (129M/M) or Ki-HuD178N mice (D178N). A-D: Transmission patterns of brain materials from the occipital lobe of sporadic Creutzfeldt-Jakob disease (sCJD) MM1 (0303) (A), thalamus of sCJD MM1+2C (I197 Th) (B), and a mixture of hippocampus and parahippocampus of sCJD MM1+2C (I197 Hip) (C), are similar to those of typical sCJD MM1 (D). E-G: By contrast, transmission patterns of brain material from the thalamus of sCJD MM1 (H89) (E) are similar to those of typical sCJD MM2T (F and $\mathbf{G})$. Data are represented as the percentage of surviving animals ( $y$ axis) plotted against the number of days after inoculation ( $x$ axis). Incubation time in Ki-Hu129M/M mice inoculated with typical sCJD MM1 material has been reported elsewhere. ${ }^{23}$ 
A

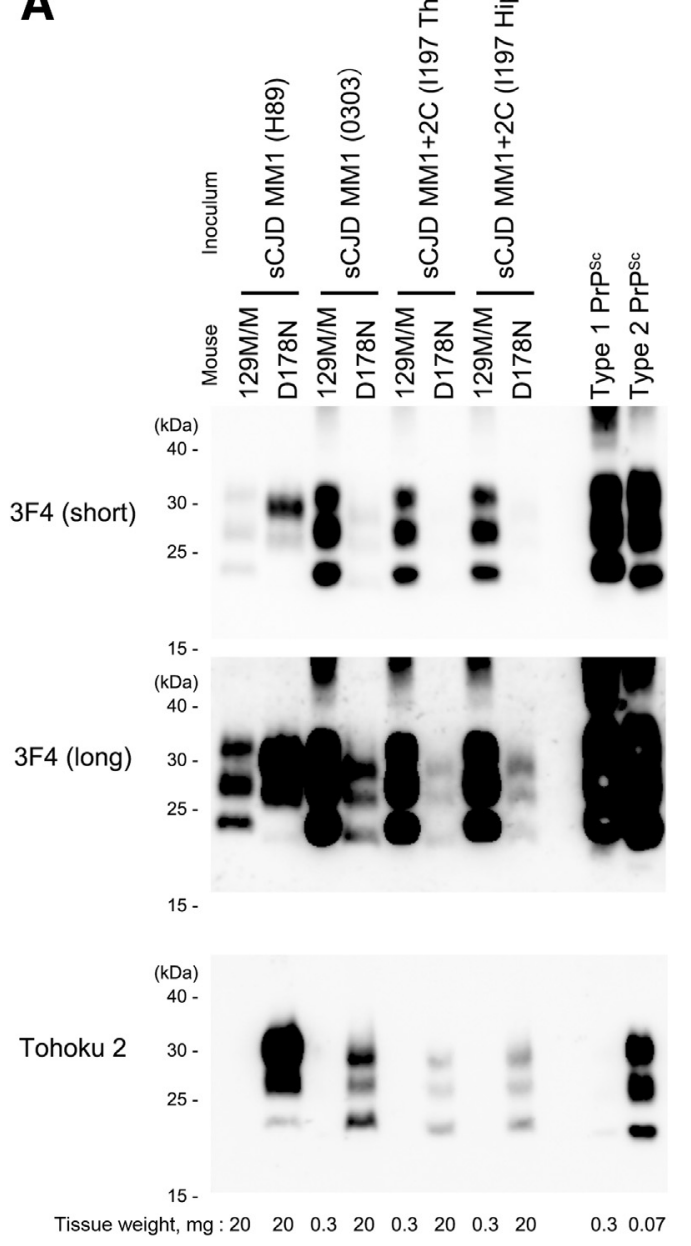

B

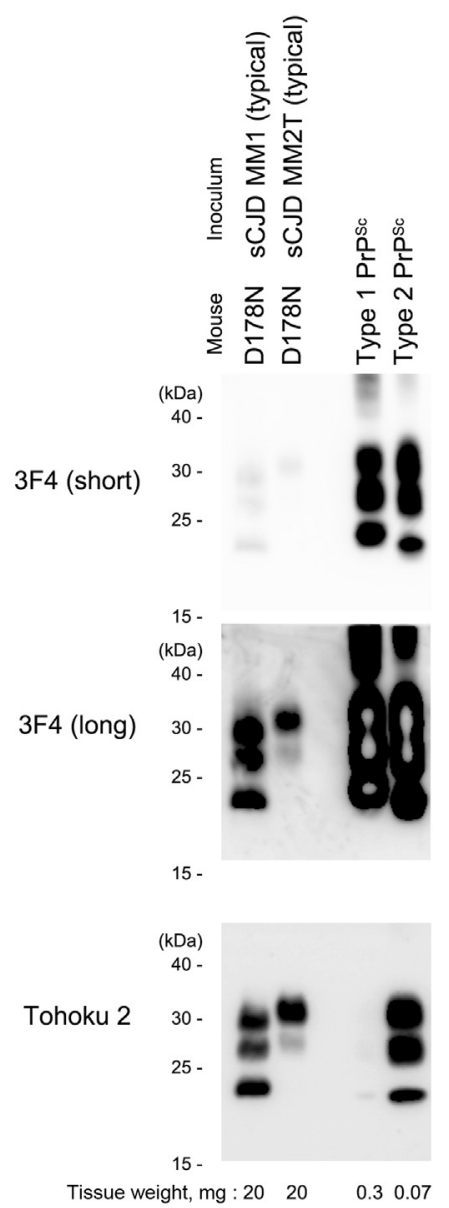

Figure 6 Biochemical properties of abnormal misfolded isoform of the prion protein $(\mathrm{PrPSC})$ in the brain of the inoculated $\mathrm{Ki}-\mathrm{Hu} 129 \mathrm{M} / \mathrm{M}$ mice (129M/M) or Ki-HuD178N mice (D178N). A and B: Western blot analysis of proteinase $\mathrm{K}$-resistant $\mathrm{PrP}^{\mathrm{Sc}}$ using the anti-PrP antibody $3 \mathrm{~F} 4$ (short and long exposures) or the type $2 \mathrm{PrP}^{\mathrm{SC}}$-specific antibody Tohoku 2. For sporadic Creutzfeldt-Jakob disease (sCJD) MM1 (0303), sCJD MM1+2C (I197 Th), and SCJD MM1+2C (I197 Hip), the inoculated Ki-Hu129M/M mice produce large amounts of type $1 \mathrm{PrP}^{\mathrm{Sc}}$ similar to those of the Ki-Hu129M/M mice inoculated with a typical SCJD MM1 material, ${ }^{23}$ and the inoculated $\mathrm{Ki}-\mathrm{HuD178N}$ mice produce a faint type $2 \mathrm{PrP}^{\mathrm{Sc}}$ similar to those of the KiHuD178N mice inoculated with the typical SCJD MM1 material. By contrast, for SCJD MM1 (H89), the inoculated $\mathrm{Ki}-\mathrm{Hu} 129 \mathrm{M} / \mathrm{M}$ mice produce a faint type $1 \mathrm{PrP} \mathrm{P}^{\mathrm{Sc}}$, and the inoculated Ki-HuD178N mice produce a diglycosylated and monoglycosylated form of dominant type $2 \mathrm{PrP}^{\mathrm{Sc}}$. The glycosylation patterns of $\mathrm{PrP}^{\mathrm{SC}}$ in the Ki-HuD178N mice inoculated with the SCJD MM1 (H89) material (A) are identical to those in the Ki-HuD178N mice inoculated with a typical SCJD MM2T material (B). The amount of brain tissue loaded in each lane is indicated beneath the lane. strain has certain infectivity to PrP-humanized mice, ${ }^{7,28,29}$ although its infectivity is lower than that of the M1 prion strain. Indeed, in the present study, one of four inocula prepared from brains in which coexistence of an M2T prion strain was suspected neuropathologically showed unique transmission properties resembling those of the M2T prion strain. Meanwhile, only transmission properties of the M1 prion strain were observed in transmission experiments of the three other inocula, suggesting that the propagation of the M2T prion strain might be overwhelmed by the predominant M1 prion strain.

The coexistence of an M2T prion strain also may affect the duration of illness of sCJD patients. In the present study, all sCJD MM1 patients with an inferior olivary degeneration showed a long duration of illness (ie, duration from onset to death), compared with the pure form of sCJD MM1. In sCJD MM/MV1+2C patients, a coexisting M2C prion strain can affect the clinical features of patients, and the duration of illness becomes longer with an increasing M2C prion strain load. ${ }^{11,12}$ The potential influence of a coexisting M2T prion strain on the clinical features, together with those on the neuropathologic, biochemical, and transmission properties, suggests that SCJD cases with a concurrent M2T prion strain should be considered as a distinctive entity such as $\mathrm{SCJD} \mathrm{MM} / \mathrm{MV} 1+2 \mathrm{~T}$ or $\mathrm{SCJD} \mathrm{MM} / \mathrm{MV} 1+2 \mathrm{C}+2 \mathrm{~T}$. The clinical features of sCJD MM/MV1+2T or sCJD MM/ $\mathrm{MV} 1+2 \mathrm{C}+2 \mathrm{~T}$ need to be clarified in the future with a larger number of patients.

In conclusion, the co-occurrence of M1 and M2T prion strains is a novel subgroup of SCJD prion strain cooccurrence. The coexisting M2T prion strain is easily and reliably detectable by histopathologic analysis of the inferior olivary nucleus of the medulla.

\section{Acknowledgments}

We thank members of the Creutzfeldt-Jakob Disease Surveillance Committee in Japan, Creutzfeldt-Jakob disease specialists in the prefectures, and Creutzfeldt-Jakob disease patients and families for providing important clinical information; Hiroko Kudo, Miyuki Yamamoto, and Ayumi Yamazaki for their excellent technical assistance; and Brent Bell for critical review of the manuscript. 


\section{Supplemental Data}

Supplemental material for this article can be found at https://doi.org/10.1016/j.ajpath.2019.02.012.

\section{References}

1. Prusiner SB, Scott MR, DeArmond SJ, Cohen FE: Prion protein biology. Cell 1998, 93:337-348

2. Colby DW, Prusiner SB: Prions. Cold Spring Harb Perspect Biol 2011, 3:a006833

3. Parchi P, Giese A, Capellari S, Brown P, Schulz-Schaeffer W, Windl O, Zerr I, Budka H, Kopp N, Piccardo P, Poser S, Rojiani A, Streichemberger N, Julien J, Vital C, Ghetti B, Gambetti P, Kretzschmar H: Classification of sporadic Creutzfeldt-Jakob disease based on molecular and phenotypic analysis of 300 subjects. Ann Neurol 1999, 46:224-233

4. Parchi P, Zou W, Wang W, Brown P, Capellari S, Ghetti B, Kopp N, Schulz-Schaeffer WJ, Kretzschmar HA, Head MW, Ironside JW, Gambetti P, Chen SG: Genetic influence on the structural variations of the abnormal prion protein. Proc Natl Acad Sci U S A 2000, 97: $10168-10172$

5. Parchi P, Strammiello R, Giese A, Kretzschmar H: Phenotypic variability of sporadic human prion disease and its molecular basis: past, present, and future. Acta Neuropathol 2011, 121:91-112

6. Bishop MT, Will RG, Manson JC: Defining sporadic CreutzfeldtJakob disease strains and their transmission properties. Proc Natl Acad Sci U S A 2010, 107:12005-12010

7. Moda F, Suardi S, Di Fede G, Indaco A, Limido L, Vimercati C, Ruggerone M, Campagnani I, Langeveld J, Terruzzi A, Brambilla A, Zerbi P, Fociani P, Bishop MT, Will RG, Manson JC, Giaccone G, Tagliavini F: MM2-thalamic Creutzfeldt-Jakob disease: neuropathological, biochemical and transmission studies identify a distinctive prion strain. Brain Pathol 2012, 22:662-669

8. Puoti G, Giaccone G, Rossi G, Canciani B, Bugiani O, Tagliavini F: Sporadic Creutzfeldt-Jakob disease: co-occurrence of different types of $\mathrm{PrP}^{\mathrm{Sc}}$ in the same brain. Neurology 1999, 53:2173-2176

9. Schoch G, Seeger H, Bogousslavsky J, Tolnay M, Janzer RC, Aguzzi A, Glatzel M: Analysis of prion strains by $\mathrm{PrP}^{\mathrm{Sc}}$ profiling in sporadic Creutzfeldt-Jakob disease. PLoS One 2006, 3:e14

10. Uro-Coste E, Cassard H, Simon S, Lugan S, Bilheude JM, PerretLiaudet A, Ironside JW, Haik S, Basset-Leobon C, Lacroux C, Peoch K, Streichenberger N, Langeveld J, Head MW, Grassi J, Hauw JJ, Schelcher F, Delisle MB, Andréoletti O: Beyond PrPres type 1/type 2 dichotomy in Creutzfeldt-Jakob disease. PLoS Pathog 2008, 4:e1000029

11. Cali I, Castellani R, Alshekhlee A, Cohen Y, Blevins J, Yuan J, Langeveld JP, Parchi P, Safar JG, Zou WQ, Gambetti P: Co-existence of scrapie prion protein types 1 and 2 in sporadic Creutzfeldt-Jakob disease: its effect on the phenotype and prion-type characteristics. Brain 2009, 132:2643-2658

12. Parchi P, Strammiello R, Notari S, Giese A, Langeveld JP, Ladogana A: Incidence and spectrum of sporadic Creutzfeldt-Jakob disease variants with mixed phenotype and co-occurrence of $\mathrm{PrP}^{\mathrm{Sc}}$ types: an updated classification. Acta Neuropathol 2009, 118: 659-671

13. Kobayashi A, Matsuura Y, Iwaki T, Iwasaki Y, Yoshida M, Takahashi H, Murayama S, Takao M, Kato S, Yamada M, Mohri S, Kitamoto T: Sporadic Creutzfeldt-Jakob disease MM1+2C and MM1 are identical in transmission properties. Brain Pathol 2016, 26:95-101
14. Kitamoto T, Ohta M, Doh-ura K, Hitoshi S, Terao Y, Tateishi J: Novel missense variants of prion protein in Creutzfeldt-Jakob disease or Gerstmann-Sträussler syndrome. Biochem Biophys Res Commun 1993, 191:709-714

15. Iwasaki $\mathrm{Y}$, Mori $\mathrm{K}$, Ito M, Mimuro M, Kitamoto T, Yoshida M: An autopsied case of MM1 + MM2-cortical with thalamic-type sporadic Creutzfeldt-Jakob disease presenting with hyperintensities on diffusion-weighted MRI before clinical onset. Neuropathology 2017, $37: 78-85$

16. Kitamoto T, Shin RW, Doh-ura K, Tomokane N, Miyazono M, Muramoto T, Tateishi J: Abnormal isoform of prion proteins accumulates in the synaptic structures of the central nervous system in patients with Creutzfeldt-Jakob disease. Am J Pathol 1992, 140: 1285-1294

17. Kitamoto T, Muramoto T, Hilbich C, Beyreuther K, Tateishi J: $\mathrm{N}$-terminal sequence of prion protein is also integrated into kuru plaques in patients with Gerstmann-Sträussler syndrome. Brain Res 1991, 545:319-321

18. Kluver H, Barrera E: A method for the combined staining of cells and fibers in the nervous system. J Neuropathol Exp Neurol 1953, 12: 400-403

19. Munesue Y, Shimazaki T, Qi Z, Isoda N, Sawa H, Aoshima K, Kimura T, Mohri S, Kitamoto T, Kobayashi A: Development of a quick bioassay for the evaluation of transmission properties of acquired prion diseases. Neurosci Lett 2018, 668:43-47

20. Grathwohl KU, Horiuchi M, Ishiguro N, Shinagawa M: Improvement of $\mathrm{PrP}^{\mathrm{Sc}}$-detection in mouse spleen early at the preclinical stage of scrapie with collagenase-completed tissue homogenization and Sarkosyl-NaCl extraction of $\mathrm{PrP}^{\mathrm{Sc}}$. Arch Virol 1996, 141:1863-1874

21. Hizume M, Kobayashi A, Teruya K, Ohashi H, Ironside JW, Mohri S, Kitamoto T: Human prion protein $(\mathrm{PrP}) 219 \mathrm{~K}$ is converted to $\operatorname{PrP}^{\mathrm{Sc}}$ but shows heterozygous inhibition in variant Creutzfeldt-Jakob disease infection. J Biol Chem 2009, 284:3603-3609

22. Asano M, Mohri S, Ironside JW, Ito M, Tamaoki N, Kitamoto T: vCJD prion acquires altered virulence through trans-species infection. Biochem Biophys Res Commun 2006, 342:293-299

23. Kobayashi A, Sakuma N, Matsuura Y, Mohri S, Aguzzi A, Kitamoto T: Experimental verification of a traceback phenomenon in prion infection. J Virol 2010, 84:3230-3238

24. Kitamoto T, Nakamura K, Nakao K, Shibuya S, Shin RW, Gondo Y, Katsuki M, Tateishi J: Humanized prion protein knock-in by Creinduced site-specific recombination in the mouse. Biochem Biophys Res Commun 1996, 222:742-747

25. Kitamoto T, Mohri S, Ironside JW, Miyoshi I, Tanaka T, Kitamoto N, Itohara S, Kasai N, Katsuki M, Higuchi J, Muramoto T, Shin RW: Follicular dendritic cell of the knock-in mouse provides a new bioassay for human prions. Biochem Biophys Res Commun 2002, 294: 280-286

26. Kanda Y: Investigation of the freely available easy-to-use software 'EZR' for medical statistics. Bone Marrow Transplant 2013, 48: 452-458

27. Parchi P, Petersen RB, Chen SG, Autilio-Gambetti L, Capellari S, Monari L, Cortelli P, Montagna P, Lugaresi E, Gambetti P: Molecular pathology of fatal familial insomnia. Brain Pathol 1998, 8:531-537

28. Korth C, Kaneko K, Groth D, Heye N, Telling G, Mastrianni J: Abbreviated incubation times for human prions in mice expressing a chimeric mouse-human prion protein transgene. Proc Natl Acad Sci U S A 2003, 100:4784-4789

29. Taguchi Y, Mohri S, Ironside JW, Muramoto T, Kitamoto T: Humanized knock-in mice expressing chimeric prion protein showed varied susceptibility to different human prions. Am J Pathol 2003, 163: $2585-2593$ 